TRANSACTIONS OF THE

AMERICAN MATHEMATICAL SOCIETY

Volume 359, Number 7, July 2007, Pages 3425-3438

S 0002-9947(07)04305-X

Article electronically published on January 26, 2007

\title{
COHOMOGENEITY ONE ACTIONS ON NONCOMPACT SYMMETRIC SPACES OF RANK ONE
}

\author{
JÜRGEN BERNDT AND HIROSHI TAMARU
}

\begin{abstract}
We classify, up to orbit equivalence, all cohomogeneity one actions on the hyperbolic planes over the complex, quaternionic and Cayley numbers, and on the complex hyperbolic spaces $\mathbb{C} H^{n}, n \geq 3$. For the quaternionic hyperbolic spaces $\mathbb{H} H^{n}, n \geq 3$, we reduce the classification problem to a problem in quaternionic linear algebra and obtain partial results. For real hyperbolic spaces, this classification problem was essentially solved by Élie Cartan.
\end{abstract}

\section{INTRODUCTION}

An isometric action on a Riemannian manifold is of cohomogeneity one if its orbit space is one-dimensional. Cohomogeneity one actions are of current interest for the construction of geometrical structures on manifolds, e.g., Einstein metrics and metrics with special holonomies. The reason is that a cohomogeneity one action can be used to reduce the system of partial differential equations describing such a geometrical structure to a nonlinear ordinary differential equation for which one might be able to find explicit solutions. Given a Riemannian manifold $M$, it is natural to find all cohomogeneity one actions on it, perhaps just up to orbit equivalence. Two cohomogeneity one actions on $M$ are orbit equivalent if there exists an isometry of $M$ that maps the orbits of one action onto the orbits of the other action. It is worthwhile to mention that the classification problem of cohomogeneity one actions up to orbit equivalence is equivalent to the classification problem of homogeneous hypersurfaces up to isometric congruence. The latter is a classical problem in submanifold theory.

The cohomogeneity one actions on spheres, equipped with their standard metric of constant curvature, have been classified by Hsiang and Lawson [14. Remarkably, any such action is orbit equivalent to the isotropy representation of a Riemannian symmetric space of rank two. For the other compact symmetric spaces of rank one the classifications were obtained by Takagi 21] for the complex projective spaces and by Iwata [15, 16] for the quaternionic projective spaces and the Cayley

Received by the editors July 12, 2005.

2000 Mathematics Subject Classification. Primary 53C35; Secondary 57S20.

Key words and phrases. Symmetric spaces, hyperbolic spaces, cohomogeneity one actions, homogeneous hypersurfaces.

The second author was partially supported by Grant-in-Aid for Young Scientists (B) 14740049 and 17740039, The Ministry of Education, Culture, Sports, Science and Technology, Japan.

(C)2007 American Mathematical Society Reverts to public domain 28 years from publication 
projective plane. For simply connected irreducible Riemannian symmetric spaces of higher rank the classification was established by Kollross [17.

The methods employed by the above authors do not work for the noncompact dual symmetric spaces. The noncompactness of the isometry group turns out to be a subtle point. There can be uncountably many families of nonisomorphic subgroups of the isometry group that act orbit equivalently by cohomogeneity one. By using the classification of isoparametric hypersurfaces on the Euclidean space $\mathbb{R}^{n}$ and the real hyperbolic space $\mathbb{R} H^{n}$ by Levi-Civita [18, Segre [20] and Cartan [10], one can obtain all cohomogeneity one actions on these spaces up to orbit equivalence. In both cases the orbit structure is either a Riemannian foliation, or a totally geodesic subspace together with the distance tubes around it. It is a general fact that a cohomogeneity one action on a symmetric space of noncompact type, or more generally on a Hadamard manifold, induces either a Riemannian foliation, or has exactly one singular orbit and the generic orbits are the distance tubes around it (see [3] for details and references).

In [4] we obtained the classification, up to orbit equivalence, of all cohomogeneity one actions on irreducible symmetric spaces of noncompact type that induce a Riemannian foliation, that is, have no singular orbit. A surprising consequence of this result is that the moduli space of all such actions just depends on the rank of the symmetric space and possible duality or triality principles on the space. In particular, on each noncompact symmetric space of rank one this moduli space consists of just two elements. The corresponding foliations are the horosphere foliation and a foliation with exactly one minimal leaf whose geometry has been investigated in [2].

The classification of all cohomogeneity one actions on irreducible symmetric spaces of noncompact type that have a totally geodesic singular orbit has been achieved in [5]. It thus remains to investigate the case of a non-totally-geodesic singular orbit. As mentioned above, in the case of $\mathbb{R}^{n}$ and $\mathbb{R} H^{n}$ a singular orbit is necessarily totally geodesic. It is remarkable that this is no longer true for the other noncompact symmetric spaces of rank one: the complex hyperbolic spaces $\mathbb{C} H^{n}(n \geq 2)$, the quaternionic hyperbolic spaces $\mathbb{H} H^{n}(n \geq 2)$, and the Cayley hyperbolic plane $\mathbb{O} H^{2}$. The first author and Brück constructed in 3 many examples of cohomogeneity one actions on these hyperbolic spaces (except for $\mathbb{C} H^{2}$ ) with a non-totally-geodesic singular orbit. The main result of this paper says that, up to orbit equivalence, there are no further cohomogeneity one actions on $\mathbb{C} H^{n}$ $(n \geq 3), \mathbb{H} H^{2}$ and $\mathbb{O} H^{2}$. We also show that every singular orbit of a cohomogeneity one action on $\mathbb{C} H^{2}$ is totally geodesic. For the quaternionic hyperbolic space $\mathbb{H} H^{n}, n \geq 3$, we prove that the set of orbit equivalence classes of cohomogeneity one actions with a singular orbit of codimension 2 is parametrized by the closed interval $[0, \pi / 2]$.

The results of this paper were partially obtained during a common visit to the Mathematical Research Institute Oberwolfach (Research in Pairs programme). We would like to thank the Institute for its support and hospitality.

\section{Preliminaries}

Let $M$ be a noncompact symmetric space of rank one. Then $M$ is either a real hyperbolic space $\mathbb{R} H^{n}$, a complex hyperbolic space $\mathbb{C} H^{n}$, a quaternionic hyperbolic space $\mathbb{H} H^{n}$, or a Cayley hyperbolic plane $\mathbb{O} H^{2}$, where $n \geq 2$. We denote by $\mathbb{F}$ one of 
the real division algebras $\mathbb{R}, \mathbb{C}, \mathbb{H}$ or $\mathbb{O}$, and by $\mathbb{F} H^{n}$ the corresponding hyperbolic space, where we assume $n=2$ if $\mathbb{F}=\mathbb{O}$. Let $G$ be the identity component of the full isometry group of $M$; that is, $G=S O^{\circ}(n, 1), S U(n, 1), S p(n, 1), F_{4}^{-20}$ for $\mathbb{F}=\mathbb{R}, \mathbb{C}, \mathbb{H}, \mathbb{O}$, respectively. We fix a point $o \in M$ and denote by $K$ the isotropy subgroup of $G$ at $o$; that is, $K=S O(n), S(U(n) U(1)), S p(n) S p(1)$, Spin(9). Then, as a homogeneous space, $M$ is isomorphic to $G / K$. We denote by $\mathfrak{g}$ and $\mathfrak{k}$ the Lie algebras of $G$ and $K$. Let $B$ be the Killing form of $\mathfrak{g}$ and $\theta$ the Cartan involution of $\mathfrak{g}$ with respect to $\mathfrak{k}$. Then $\langle X, Y\rangle=-B(X, \theta Y)$ is a positive definite inner product on $\mathfrak{g}$. Let $\mathfrak{g}=\mathfrak{k}+\mathfrak{p}$ be the Cartan decomposition of $\mathfrak{g}$ induced by $\theta$. The restriction of $\langle\cdot, \cdot\rangle$ to $\mathfrak{p}$ induces a Riemannian metric on $G / K$ turning it into a Riemannian symmetric space of rank one. We normalize the Riemannian metric on $M$ so that it becomes isometric to $G / K$ with the induced metric we just described.

Let $\mathfrak{a}$ be a maximal abelian subspace of $\mathfrak{p}$, which is just a one-dimensional linear subspace since the rank of $M$ is one, and let

$$
\mathfrak{g}=\mathfrak{g}_{-2 \alpha}+\mathfrak{g}_{\alpha}+\mathfrak{g}_{0}+\mathfrak{g}_{\alpha}+\mathfrak{g}_{2 \alpha}
$$

be the corresponding restricted root space decomposition of $\mathfrak{g}$. Note that $\mathfrak{g}_{-2 \alpha}$ and $\mathfrak{g}_{2 \alpha}$ are trivial if $\mathbb{F}=\mathbb{R}$. Then

$$
\mathfrak{g}=\mathfrak{k}+\mathfrak{a}+\mathfrak{n} \quad \text { with } \quad \mathfrak{n}=\mathfrak{g}_{\alpha}+\mathfrak{g}_{2 \alpha}
$$

is an Iwasawa decomposition of $\mathfrak{g}$. The subalgebra $\mathfrak{n}$ of $\mathfrak{g}$ is abelian if $\mathbb{F}=\mathbb{R}$ and two-step nilpotent otherwise. In fact, $\mathfrak{n}$ is isomorphic to the $(2 n-1)$-dimensional Heisenberg algebra if $\mathbb{F}=\mathbb{C}$, and to a certain generalized Heisenberg algebra if $\mathbb{F} \in\{\mathbb{H}, \mathbb{O}\}$ (see $\left[\underline{6}\right.$ for more details on this). Moreover, $\mathfrak{z}=\mathfrak{g}_{2 \alpha}$ is the center of $\mathfrak{n}$ and equal to the derived subalgebra $[\mathfrak{n}, \mathfrak{n}]$ of $\mathfrak{n}$. The dimension of $\mathfrak{z}$ is equal to $1,3,7$ for $\mathbb{F}=\mathbb{C}, \mathbb{H}, \mathbb{O}$, respectively. The subalgebra $\mathfrak{a}+\mathfrak{n}$ of $\mathfrak{g}$ is solvable and $\mathfrak{n}$ is the derived subalgebra of $\mathfrak{a}+\mathfrak{n}$.

We denote by $A$ resp. $N$ the connected closed subgroup of $G$ with Lie algebra $\mathfrak{a}$ resp. $\mathfrak{n}$. Then $G=K A N$ is an Iwasawa decomposition of $G$ and, since $K$ is the isotropy subgroup of $G$ at $o$, the solvable subgroup $A N$ of $G$ acts simply transitively on $M$. Thus $M$ is isometric to the solvable Lie group $A N$ equipped with a suitable left-invariant Riemannian metric.

We define $\mathfrak{v}=\mathfrak{g}_{\alpha}$. Then we can identify $\mathfrak{v}$ with $\mathbb{R}^{n-1}, \mathbb{C}^{n-1}, \mathbb{H}^{n-1}, \mathbb{O}$ for $\mathbb{F}=$ $\mathbb{R}, \mathbb{C}, \mathbb{H}, \mathbb{O}$, respectively. More precisely, if $\mathbb{F}=\mathbb{R}$, then $\mathfrak{v}$ is isomorphic to $\mathbb{R}^{n-1}$ as a real vector space. If $\mathbb{F}=\mathbb{C}$, the Kähler structure on $\mathbb{C} H^{n}$ induces a complex vector space structure on $\mathfrak{v}$ so that it becomes isomorphic to $\mathbb{C}^{n-1}$, and if $\mathbb{F}=\mathbb{H}$, the quaternionic Kähler structure on $\mathbb{H} H^{n}$ induces a (right) quaternionic vector space structure on $\mathfrak{v}$ so that it becomes isomorphic to the (right) quaternionic vector space $\mathbb{H}^{n-1}$. For $\mathbb{F}=\mathbb{O}$ we simply identify $\mathbb{O}$ with $\mathbb{R}^{8}$, and $\mathfrak{v}$ becomes isomorphic to $\mathbb{O}$ as a real vector space.

\section{The REDUCTION}

In this section we reduce our classification problem to the problem of classifying certain subalgebras of $\mathfrak{a}+\mathfrak{n}$. We start with a general result about cohomogeneity one actions on Hadamard manifolds, i.e., connected, simply connected, complete Riemannian manifolds of nonpositive curvature. Every symmetric space of noncompact type is a Hadamard manifold. We recall that Cartan's Fixed Point Theorem states that the action of any compact subgroup of the isometry group of a Hadamard 
manifold has a fixed point. We refer to [12] for more details on Hadamard manifolds and Cartan's Fixed Point Theorem.

Proposition 3.1. Let $M$ be a Hadamard manifold and $H$ a connected subgroup of the isometry group of $M$ that acts with cohomogeneity one on $M$ and has a singular orbit $F$. Then there exists a connected solvable subgroup of $H$ that acts transitively on $F$.

Proof. We choose a Levi-Malcev decomposition

$$
\mathfrak{h}=\mathfrak{h}_{\text {ss }}+\mathfrak{h}_{\text {solv }}
$$

of the Lie algebra $\mathfrak{h}$ of $H$ into the semidirect sum of a semisimple subalgebra $\mathfrak{h}_{s s}$ and a solvable ideal $\mathfrak{h}_{\text {solv }}$. For the semisimple subalgebra $\mathfrak{h}_{s s}$ we choose an Iwasawa decomposition

$$
\mathfrak{h}_{s s}=\mathfrak{h}_{s s}^{c p c t}+\mathfrak{h}_{s s}^{\text {solv }}
$$

of $\mathfrak{h}_{s s}$ into the vector space direct sum of a compact subalgebra $\mathfrak{h}_{s s}^{c p c t}$ and a solvable subalgebra $\mathfrak{h}_{s s}^{\text {solv. }}$. Then

$$
\mathfrak{h}=\mathfrak{h}_{s s}^{\text {cpct }}+\left(\mathfrak{h}_{s s}^{\text {solv }}+\mathfrak{h}_{\text {solv }}\right)
$$

where $\mathfrak{h}_{s s}^{\text {solv }}+\mathfrak{h}_{\text {solv }}$ is a semidirect sum of the two solvable subalgebras so that $\mathfrak{h}_{\text {solv }}$ is the ideal in it. Note that the semidirect sum of two solvable Lie algebras is solvable as well. We denote by $H_{s s}^{c p c t}$ and $H^{\text {solv }}$ the connected subgroup of $H$ with Lie algebra $\mathfrak{h}_{s s}^{c p c t}$ and $\mathfrak{h}_{s s}^{\text {solv }}+\mathfrak{h}_{\text {solv }}$, respectively.

By Cartan's Fixed Point Theorem, there exists a point $p \in M$ that is fixed under the action of the compact group $H_{s s}^{c p c t}$. If $p \in F$, then clearly the solvable group $H^{\text {solv }}$ acts transitively on $F$. If $p \notin F$, then $p$ is on a principal orbit of the $H$-action on $M$, and it follows that the solvable group $H^{\text {solv }}$ acts transitively on this principal orbit. Since the action of $H$ on $M$ is of cohomogeneity one, we easily see that $H^{\text {solv }}$ acts transitively on each orbit of the $H$-action and, in particular, also transitively on the singular orbit $F$. This finishes the proof of Proposition 3.1 .

We denote by $M(\infty)$ the ideal boundary of $M$ whose points are given by the equivalence classes of asymptotic geodesics in $M$, and equip $\bar{M}=M \cup M(\infty)$ with the cone topology. The action of $H$ on $M$ extends canonically to an action of $H$ on $\bar{M}$.

From now on we assume that $M=\mathbb{F} H^{n}$ and that the singular orbit $F$ of the cohomogeneity one action by $H$ on $M$ is not totally geodesic. Then none of the $H$-orbits on $M$ is totally geodesic, and a result by Alekseevsky and Di Scala [1] implies that there exists a unique point $x \in M(\infty)$ that is fixed under the $H$-action on $M(\infty)$. We fix a point $o \in F$ and consider the Iwasawa decomposition

$$
\mathfrak{g}=\mathfrak{k}+\mathfrak{a}+\mathfrak{n}
$$

that is determined by $o$ and $x$. Since $H \cdot x=x$, we have

$$
\mathfrak{h} \subset \mathfrak{k}_{x}+\mathfrak{a}+\mathfrak{n},
$$

where $\mathfrak{k}_{x}$ is the centralizer of $\mathfrak{a}$ in $\mathfrak{k}$. We denote by $K_{x}$ the connected subgroup of $K$ with Lie algebra $\mathfrak{k}_{x}$. Then we have

$$
K_{x}=S O(n-1), S(U(n-1) U(1)), S p(n-1) \operatorname{Sp}(1), \operatorname{Spin}(7)
$$

for $\mathbb{F}=\mathbb{R}, \mathbb{C}, \mathbb{H}, \mathbb{O}$, respectively, and $H \subset K_{x} A N$.

By Proposition 3.1 there exists a solvable subgroup $S$ of $H$ that acts transitively on the singular orbit $F$. We denote by $\mathfrak{s}$ the subalgebra of $\mathfrak{h}$ corresponding to $S \subset H$. 
We recall that we may write the nilpotent subalgebra $\mathfrak{n}$ in the form $\mathfrak{n}=\mathfrak{g}_{\alpha}+\mathfrak{g}_{2 \alpha}$ with some suitable root spaces $\mathfrak{g}_{\alpha}$ and $\mathfrak{g}_{2 \alpha}$. Since $\mathfrak{k}_{x}$ centralizes $\mathfrak{a}$, it normalizes each root space and hence $\mathfrak{n}$, which implies that $\mathfrak{a}+\mathfrak{n}$ is an ideal in $\mathfrak{k}_{x}+\mathfrak{a}+\mathfrak{n}$. Thus the canonical projection

$$
\pi: \mathfrak{k}_{x}+\mathfrak{a}+\mathfrak{n} \rightarrow \mathfrak{k}_{x}
$$

is a Lie algebra homomorphism, and it follows that

$$
\mathfrak{s}_{c}=\pi(\mathfrak{s})
$$

is a solvable subalgebra of $\mathfrak{k}_{x}$. Since every solvable subalgebra of a compact Lie algebra is abelian, we conclude that

$$
\mathfrak{s}_{c} \text { is an abelian subalgebra of } \mathfrak{k}_{x} .
$$

Let

$$
\tau: \mathfrak{k}_{x}+\mathfrak{a}+\mathfrak{n} \rightarrow \mathfrak{a}+\mathfrak{n}
$$

be the canonical projection and define

$$
\mathfrak{s}_{n}=\tau(\mathfrak{s}) .
$$

It is clear that

$$
\operatorname{dim} \mathfrak{s}_{n}=\operatorname{dim} F .
$$

Our aim is to show that $\mathfrak{s}_{n}$ is a subalgebra of $\mathfrak{a}+\mathfrak{n}$ and that the orbit through $o$ of the action of the corresponding subgroup $S_{n}$ of $A N$ is just the singular orbit $F$. For each $k \in K_{x}$ the differential $d_{o} k$ of $k$ at $o$ is given by $d_{o} k=\operatorname{Ad}(k) \mid(\mathfrak{a}+\mathfrak{n})$, where we identify $T_{o} M$ with $\mathfrak{a}+\mathfrak{n}$ by means of $M=G / K=A N$. Since the isotropy subgroup $H_{o}$ of $H$ at $o$ acts transitively on the unit sphere in the normal space $\nu_{o} F$ of $F$ at $o$, and as $\mathfrak{k}_{x}$ centralizes $\mathfrak{a}$, we necessarily have

$$
\nu_{o} F \subset \mathfrak{n}
$$

and hence

$$
\mathfrak{a} \subset \mathfrak{s}_{n} .
$$

We shall now prove that

$$
[\mathfrak{s}, \mathfrak{s}]=\mathfrak{s}_{n} \cap \mathfrak{n} .
$$

Since $\mathfrak{s} \subset \mathfrak{s}_{c}+\mathfrak{s}_{n}$, we have

$$
[\mathfrak{s}, \mathfrak{s}] \subset\left[\mathfrak{s}_{c}, \mathfrak{s}_{c}\right]+\left[\mathfrak{s}_{c}, \mathfrak{s}_{n}\right]+\left[\mathfrak{s}_{n}, \mathfrak{s}_{n}\right] .
$$

The subalgebra $\left[\mathfrak{s}_{c}, \mathfrak{s}_{c}\right]$ is trivial since $\mathfrak{s}_{c}$ is abelian according to (3.1). Since $\mathfrak{s}_{c} \subset \mathfrak{k}_{x}$, $\mathfrak{s}_{n} \subset \mathfrak{a}+\mathfrak{n}$ and $\mathfrak{k}_{x}$ centralizes $\mathfrak{a}$ and normalizes $\mathfrak{n}$, we have $\left[\mathfrak{s}_{c}, \mathfrak{s}_{n}\right] \subset\left[\mathfrak{k}_{x}, \mathfrak{a}+\mathfrak{n}\right] \subset \mathfrak{n}$. Finally, since $\mathfrak{s}_{n} \subset \mathfrak{a}+\mathfrak{n}$ and $\mathfrak{n}$ is the derived subalgebra of $\mathfrak{a}+\mathfrak{n}$, we see that $\left[\mathfrak{s}_{n}, \mathfrak{s}_{n}\right] \subset \mathfrak{n}$. Altogether this implies $[\mathfrak{s}, \mathfrak{s}] \subset \mathfrak{n}$, which readily yields $[\mathfrak{s}, \mathfrak{s}] \subset \mathfrak{s}_{n} \cap \mathfrak{n}$. For the converse, we fix the element $B \in \mathfrak{a}$ for which $[B, V]=V$ and $[B, Z]=2 Z$ holds for all $V \in \mathfrak{v}=\mathfrak{g}_{\alpha}$ and $Z \in \mathfrak{z}=\mathfrak{g}_{2 \alpha}$. Because of (3.4) there exists an element $\tilde{B} \in \mathfrak{s}_{c}$ so that $\tilde{B}+B \in \mathfrak{s}$. Let $X=V+Z \in \mathfrak{v}+\mathfrak{z}$ be an arbitrary element in the orthogonal complement of $[\mathfrak{s}, \mathfrak{s}]$ in $\mathfrak{s}_{n} \cap \mathfrak{n}$. Then there exists a vector $\tilde{X} \in \mathfrak{s}_{c}$ so that $\tilde{X}+X \in \mathfrak{s}$, and we have

$$
0=\langle X,[\tilde{B}+B, \tilde{X}+X]\rangle=\langle X,[\tilde{B}, \tilde{X}]+[\tilde{B}, X]+[B, \tilde{X}]+[B, X]\rangle .
$$


Since $\mathfrak{s}_{c}$ is abelian we have $[\tilde{B}, \tilde{X}]=0$. Since $\operatorname{ad}(\tilde{B})$ is a skewsymmetric transformation we have $\langle X,[\tilde{B}, X]\rangle=0$, and since $\mathfrak{s}_{c} \subset \mathfrak{k}_{x}$ and $\mathfrak{k}_{x}$ centralizes $\mathfrak{a}$ we have $[B, \tilde{X}]=0$. This implies

$$
0=\langle X,[B, X]\rangle=\langle V+Z, V+2 Z\rangle=\langle V, V\rangle+2\langle Z, Z\rangle,
$$

and hence $V=0=Z$. Thus $X=0$, which implies that the orthogonal complement of $[\mathfrak{s}, \mathfrak{s}]$ in $\mathfrak{s}_{n} \cap \mathfrak{n}$ is trivial. This establishes the proof of (3.5).

Our next aim is to prove that

$$
\nu_{o} F \subset \mathfrak{v}=\mathfrak{g}_{\alpha} .
$$

From (3.3) we already know that $\nu_{o} F \subset \mathfrak{n}$. If $\nu_{o} F \cap \mathfrak{v} \neq 0$, we readily get $\nu_{o} F \subset \mathfrak{v}$, because $\operatorname{Ad}\left(H_{o}\right)$ acts transitively on the unit sphere in $\nu_{o} F$ and preserves $\mathfrak{v}$. Now assume that $\nu_{o} F \cap \mathfrak{v}=0$. Then (3.5) implies that the canonical projection of $[\mathfrak{s}, \mathfrak{s}] \subset \mathfrak{v}+\mathfrak{z}$ onto $\mathfrak{v}$ is the entire space $\mathfrak{v}$. Thus, for each $V \in \mathfrak{v}$ there exists an element $V^{\prime} \in \mathfrak{z}$ so that $V+V^{\prime} \in[\mathfrak{s}, \mathfrak{s}]$. Since $[\mathfrak{s}, \mathfrak{s}]$ is a subalgebra, we get

$$
[V, W]=\left[V+V^{\prime}, W+W^{\prime}\right] \in[\mathfrak{s}, \mathfrak{s}]
$$

for all $V, W \in \mathfrak{v}$. But since $[\mathfrak{v}, \mathfrak{v}]=\left[\mathfrak{g}_{\alpha}, \mathfrak{g}_{\alpha}\right]=\mathfrak{g}_{2 \alpha}=\mathfrak{z}$, this implies $\mathfrak{z} \subset[\mathfrak{s}, \mathfrak{s}]$ and hence $\nu_{o} F \subset \mathfrak{v}$. This establishes the proof of (3.6).

From (3.6) we see that there exists a linear subspace $\mathfrak{v}_{o}$ of $\mathfrak{v}$ so that $\mathfrak{s}_{n}=\mathfrak{a}+\mathfrak{v}_{o}+\mathfrak{z}$. Using the Lie algebra structure of $\mathfrak{a}+\mathfrak{n}$, we get:

$$
\mathfrak{s}_{n} \text { is a subalgebra of } \mathfrak{a}+\mathfrak{n} .
$$

Let $S_{n}$ be the connected subgroup of $A N$ with Lie algebra $\mathfrak{s}_{n}$. Our next aim is to show that the orbit $S_{n} \cdot o$ of $S_{n}$ through $o$ coincides with the singular orbit $F$. For this purpose we define

$$
\mathfrak{t}=\mathfrak{k}_{x} \cap \mathfrak{s} \subset \mathfrak{s}_{c} \text { and } \quad \mathfrak{s}^{\prime}=\mathbb{R}(\tilde{B}+B)+[\mathfrak{s}, \mathfrak{s}],
$$

where $B \in \mathfrak{a}$ and $\tilde{B} \in \mathfrak{s}_{c}$ are defined as above. Since $\mathfrak{t} \subset \mathfrak{s}_{c}, \tilde{B} \in \mathfrak{s}_{c}$ and $\mathfrak{s}_{c}$ is abelian we have $[\mathfrak{t}, \tilde{B}]=0$, and since $\mathfrak{t} \subset \mathfrak{k}_{x}$ and $\mathfrak{k}_{x}$ centralizes $\mathfrak{a}$ we have $[\mathfrak{t}, B]=0$. Clearly, we also have $[\mathfrak{t},[\mathfrak{s}, \mathfrak{s}]] \subset[\mathfrak{s}, \mathfrak{s}] \subset \mathfrak{s}^{\prime}$ since $\mathfrak{t} \subset \mathfrak{s}$ and $[\mathfrak{s}, \mathfrak{s}] \subset \mathfrak{s}$. Altogether this implies $\left[\mathfrak{t}, \mathfrak{s}^{\prime}\right] \subset \mathfrak{s}^{\prime}$. Moreover, since $\mathfrak{s}^{\prime} \subset \mathfrak{s}$, we have $\left[\mathfrak{s}^{\prime}, \mathfrak{s}^{\prime}\right] \subset[\mathfrak{s}, \mathfrak{s}] \subset \mathfrak{s}^{\prime}$, which shows that $\mathfrak{s}^{\prime}$ is a subalgebra of $\mathfrak{s}$. It follows that $\mathfrak{s}^{\prime}$ is an ideal in $\mathfrak{s}$ and $\mathfrak{s}=\mathfrak{t}+\mathfrak{s}^{\prime}$ (semidirect sum). Let $S^{\prime}$ be the connected subgroup of $S$ with Lie algebra $\mathfrak{s}^{\prime}$. Since $\mathfrak{t} \subset \mathfrak{h}_{o}$ we see that $S^{\prime}$ acts transitively on $F$, i.e., $S^{\prime} \cdot o=F$. For all $V+Z \in[\mathfrak{s}, \mathfrak{s}] \subset \mathfrak{n}=\mathfrak{v}+\mathfrak{z}$ we have

$$
[\tilde{B}, V+Z]+V+2 Z=[\tilde{B}, V+Z]+[B, V+Z]=[\tilde{B}+B, V+Z] \in \mathfrak{s}^{\prime}
$$

since $\mathfrak{s}^{\prime}$ is a subalgebra. But $A$ and $V+Z$ are in $\mathfrak{s}_{n}$ according to (3.4) and (3.5), and since $\mathfrak{s}_{n}$ is a subalgebra by (3.7), we have $V+2 Z=[B, V+Z] \in \mathfrak{s}_{n} \cap \mathfrak{n}=[\mathfrak{s}, \mathfrak{s}] \subset \mathfrak{s}^{\prime}$ by (3.5). By (3.8) this implies $[\tilde{B}, V+Z] \in \mathfrak{s}^{\prime}$. But $\tilde{B} \in \mathfrak{s}_{c} \subset \mathfrak{k}_{x}$ and thus ad( $(\tilde{B})$ leaves $\mathfrak{v}$ and $\mathfrak{z}$ invariant, which implies that $[\tilde{B}, V+Z] \in \mathfrak{s}^{\prime} \cap \mathfrak{n} \subset[\mathfrak{s}, \mathfrak{s}] \subset \mathfrak{s}_{n}$. We thus have proved that $\mathfrak{s}_{n}$ is normalized by $\tilde{B}$, i.e., $\left[\tilde{B}, \mathfrak{s}_{n}\right] \subset \mathfrak{s}_{n}$. Let Exp be the Lie exponential map of $\mathfrak{g}$. We now get

$$
F=S^{\prime} \cdot o \subset \operatorname{Exp}(\mathbb{R} \tilde{B}) S_{n} \cdot o=S_{n} \operatorname{Exp}(\mathbb{R} \tilde{B}) \cdot o=S_{n} \cdot o
$$

since $\operatorname{Exp}(\mathbb{R} \tilde{B})$ normalizes $S_{n}$ and $\operatorname{Exp}(\mathbb{R} \tilde{B}) \cdot o \subset H_{o} \cdot o=o$. Finally, by (3.2) the 
dimensions of $F$ and $S_{n}$ coincide, and since both $F$ and $S_{n} \cdot o$ are complete, we must have $F=S_{n} \cdot o$. We thus have proved:

Theorem 3.2. Let $H$ be a connected subgroup of $G=I^{o}\left(\mathbb{F} H^{n}\right)$ that acts on $\mathbb{F} H^{n}$ with cohomogeneity one and with a non-totally-geodesic singular orbit $F$. Then there exists a unique point $x \in M(\infty)$ that is fixed under the induced action of $H$ on $M(\infty)$. Let $o \in F, K$ the isotropy group of $G$ at o, and $\mathfrak{g}=\mathfrak{k}+\mathfrak{a}+\mathfrak{n}$ the Iwasawa decomposition of $\mathfrak{g}$ that is induced by $o$ and $x$. Then there exists a subalgebra $\mathfrak{s}$ of $\mathfrak{a}+\mathfrak{n}$ of the form $\mathfrak{s}=\mathfrak{a}+\mathfrak{v}_{o}+\mathfrak{z}$ with some linear subspace $\mathfrak{v}_{o}$ of $\mathfrak{v}$, so that $F$ is the orbit of the connected subgroup $S$ of AN with Lie algebra $\mathfrak{s}$.

\section{The Classification}

In this section we discuss the classification of cohomogeneity one actions on noncompact symmetric spaces of rank one up to orbit equivalence. Recall that such an action has either no singular orbit or exactly one singular orbit.

No singular orbit. In [4] it was shown that there exist only two such actions without a singular orbit. The first one is given by the action of the nilpotent group $N$ in an Iwasawa decomposition $G=K A N$ of $G=I^{o}\left(\mathbb{F} H^{n}\right)$, and the orbits form a horosphere foliation. The second one is given by the subgroup $S$ of $A N$ with Lie algebra $\mathfrak{s}=\mathfrak{a}+\mathfrak{v}_{o}+\mathfrak{z}$, where $\mathfrak{v}_{O}$ is a linear subspace of $\mathfrak{v}$ with codimension one. The corresponding foliation has exactly one minimal leaf and has been investigated in detail in [2]. In the case of $\mathbb{R} H^{n}$ the minimal leaf is a totally geodesic $\mathbb{R} H^{n-1} \subset \mathbb{R} H^{n}$.

Totally geodesic singular orbit. The cohomogeneity one actions on $\mathbb{F} H^{n}$ with a totally geodesic singular orbit $F$ are given by:

$$
\begin{aligned}
& M=\mathbb{R} H^{n}: F \in\left\{\mathrm{pt}, \mathbb{R} H^{1}, \ldots, \mathbb{R} H^{n-2}\right\} ; \\
& M=\mathbb{C} H^{n}: F \in\left\{\mathrm{pt}, \mathbb{C} H^{1}, \ldots, \mathbb{C} H^{n-1}, \mathbb{R} H^{n}\right\} ; \\
& M=\mathbb{H} H^{n}: F \in\left\{\mathrm{pt}, \mathbb{H} H^{1}, \ldots, \mathbb{H} H^{n-1}, \mathbb{C} H^{n}\right\} ; \\
& M=\mathbb{O} H^{2}: F \in\left\{\mathrm{pt}, \mathbb{O} H^{1}, \mathbb{H} H^{2}\right\} .
\end{aligned}
$$

Here, pt is a point in $\mathbb{F} H^{n}$, and the corresponding cohomogeneity one action is just the action of the isotropy group of $I^{o}\left(\mathbb{F} H^{n}\right)$ at that point. More details about this can be found in 3 .

Non-totally-geodesic singular orbit. We now come to the classification of cohomogeneity one actions with a non-totally-geodesic singular orbit $F$. We will use the same notation as in the previous section. Let $H$ be the connected component of the group of isometries of $M$ that leave $F$ invariant. By Theorem 3.2 there exists a unique point $x \in M(\infty)$ that is fixed under the induced action of $H$ on $M(\infty)$. Let $o \in F, K$ the isotropy group of $G$ at $o$, and $\mathfrak{g}=\mathfrak{k}+\mathfrak{a}+\mathfrak{n}$ the Iwasawa decomposition of $\mathfrak{g}$ that is induced by $o$ and $x$. Using again Theorem 3.2, there exists a subalgebra $\mathfrak{s}$ of $\mathfrak{a}+\mathfrak{n}$ of the form $\mathfrak{s}=\mathfrak{a}+\mathfrak{v}_{o}+\mathfrak{z}$ with some linear subspace $\mathfrak{v}_{o}$ of $\mathfrak{v}$, so that $F$ is the orbit of the connected subgroup $S$ of $A N$ with Lie algebra $\mathfrak{s}$. From the construction it is clear that the identity component of $H_{o}$ coincides with the identity component $N_{K}^{o}(\mathfrak{s})$ of the normalizer $N_{K}(\mathfrak{s})$ of $\mathfrak{s}$ in $K$. In order that $H$ acts with cohomogeneity one it is therefore necessary and sufficient that the action of $N_{K}^{o}(\mathfrak{s})$ on the normal space $\nu_{o} F$ is transitive on the unit sphere in $\nu_{o} F$. Note that $N_{K}^{o}(\mathfrak{s}) \subset K_{x}$. Since all Iwasawa decompositions of $\mathfrak{g}$ are conjugate to each other under an inner automorphism of $\mathfrak{g}$, it therefore remains to classify all subalgebras 
$\mathfrak{s}$ of $\mathfrak{a}+\mathfrak{n}$ of the form $\mathfrak{s}=\mathfrak{a}+\mathfrak{v}_{o}+\mathfrak{z}$ with some linear subspace $\mathfrak{v}_{o}$ of $\mathfrak{v}$ such that $N_{K}^{o}(\mathfrak{s})$ acts transitively on the unit sphere in $\mathfrak{v}_{o}^{\perp}$, the orthogonal complement of $\mathfrak{v}_{O}$ in $\mathfrak{v}$. This proves the first part of the following theorem:

Theorem 4.1. Let $\mathfrak{g}=\mathfrak{k}+\mathfrak{a}+\mathfrak{n}$ be the Iwasawa decomposition induced by $o \in M$ and $x \in M(\infty)$.

(i) Let $\mathfrak{v}_{o}$ be a linear subspace of $\mathfrak{v}$ so that $\operatorname{dim} \mathfrak{v}_{o}^{\perp} \geq 2$ and $N_{K_{x}}^{o}\left(\mathfrak{v}_{o}\right)$ acts transitively on the unit sphere in $\mathfrak{v}_{o}^{\perp}$. Then the connected subgroup of $G$ with Lie algebra $N_{\mathfrak{k}_{x}}^{o}\left(\mathfrak{v}_{o}\right)+\mathfrak{a}+\mathfrak{v}_{o}+\mathfrak{z}$ acts on $M$ with cohomogeneity one so that the orbit through o is singular. Furthermore, every cohomogeneity one action on $M$ with a non-totally-geodesic singular orbit can be obtained in this way up to orbit equivalence.

(ii) Let $\mathfrak{v}_{o}$ and $\mathfrak{v}_{o}^{\prime}$ be linear subspaces of $\mathfrak{v}$ as in (i), and assume that the corresponding cohomogeneity one actions have non-totally-geodesic singular orbits. Then, these actions are orbit equivalent if and only if there exists an isometry $k \in K_{x}$ so that $\operatorname{Ad}(k) \mathfrak{v}_{o}=\mathfrak{v}_{o}^{\prime}$.

Proof. It remains to prove part (ii). The "if"-part of the statement is obvious. Conversely, assume that the two cohomogeneity one actions are orbit equivalent. Then the corresponding singular orbits, say $S$ and $S^{\prime}$, are congruent under an isometry $k$ of $M$. We may assume that $k$ fixes $o$. By construction, the normalizers $N_{G}(S)$ and $N_{G}\left(S^{\prime}\right)$ fix $x$, the point at infinity that determines our Iwasawa decomposition. Then $k$ must fix $x$ as well, since $k N_{G}(S) k^{-1}=N_{G}\left(S^{\prime}\right)$ and $x$ is the unique fixed point in $M(\infty)$ of $N_{G}(S)$ and of $N_{G}\left(S^{\prime}\right)$. Therefore we conclude that $\operatorname{Ad}(k) \mathfrak{v}_{o}=\mathfrak{v}_{o}^{\prime}$.

We now discuss the four different hyperbolic spaces individually.

$M=\mathbb{R} H^{n}$ It follows from the classification of isoparametric hypersurfaces in $\mathbb{R} H^{n}$ by Cartan [10] that there exist no such actions. Since a singular orbit of a cohomogeneity one action is necessarily minimal, one can also apply a result by Di Scala and Olmos [1] stating that every minimal homogeneous submanifold of $\mathbb{R} H^{n}$ is totally geodesic.

The classification also follows easily from Theorem 4.1 Assume there is a cohomogeneity one action on $\mathbb{R} H^{n}$ with a non-totally-geodesic singular orbit $F$. Theorem 4.1 implies that the action is orbit equivalent to the $H$-action induced from $\mathfrak{h}=N_{\mathfrak{k}_{x}}^{o}\left(\mathfrak{v}_{o}\right)+\mathfrak{a}+\mathfrak{v}_{o}$ for some suitable subspace $\mathfrak{v}_{o}$ of $\mathfrak{v}$. But for such an $H$-action the orbit $F=H \cdot o$ is totally geodesic, which is a contradiction.

$M=\mathbb{C} H^{n}$ In this case the Kähler structure on $\mathbb{C} H^{n}$ induces a complex structure $J$ on $\mathfrak{v}$ so that $\mathfrak{v}$ is isomorphic to $\mathbb{C}^{n-1}$ as a complex vector subspace. Let $\mathfrak{v}_{o}$ be a linear subspace of $\mathfrak{v}$ so that $\operatorname{dim}_{\mathbb{R}} \mathfrak{v}_{o}^{\perp} \geq 2$. Recall that the Kähler angle of a nonzero vector $v \in \mathfrak{v}_{o}^{\perp} \subset \mathbb{C}^{n-1}$ is defined as the angle between $J v$ and $\mathfrak{v}_{o}^{\perp}$. In order that $N_{K}^{o}(\mathfrak{s})$ acts transitively on the unit sphere in $\mathfrak{v}_{o}^{\perp}$ it is necessary that the Kähler angle of $\mathfrak{v}_{o}^{\perp}$ does not depend on the choice of the unit vector in $\mathfrak{v}_{O}^{\perp}$. We thus assume that for all nonzero vectors $v \in \mathfrak{v}_{o}^{\perp}$ the Kähler angle is equal to some $\varphi \in[0, \pi / 2]$. In the special case that $\varphi=0, \mathfrak{v}_{o}^{\perp}$ is a complex subspace of $\mathfrak{v}$, and if $\varphi=\pi / 2$, then $\mathfrak{v}_{o}^{\perp}$ is a real subspace of $\mathfrak{v}$. The subspaces of complex vector spaces with constant Kähler angle have been classified in [3]. For $\varphi=0$ we just have the complex subspaces and for $\varphi=\pi / 2$ the real subspaces, and in both cases the congruence classes (under the action of $K_{x}=U(n-1)$ on $\mathfrak{v}=\mathbb{C}^{n-1}$ ) are parametrized by the complex, resp. real, dimension. For $\varphi \in(0, \pi / 2)$ there exists exactly one congruence class 
of subspaces with constant Kähler angle $\varphi$ for each dimension $0<2 k \leq n-1$. For any such subspace the resulting action on $\mathbb{C} H^{n}$ is of cohomogeneity one and $F$ is a non-totally-geodesic singular orbit unless $\varphi=0$ (then $F$ is a totally geodesic complex submanifold). Using Theorem 4.1 we therefore conclude:

Theorem 4.2. The moduli space of all cohomogeneity one actions on $\mathbb{C} H^{n}, n \geq 2$, with a non-totally-geodesic singular orbit (up to orbit equivalence) is isomorphic to the disjoint union

$$
\{2, \ldots, n-1\} \cup((0, \pi / 2) \times\{2 k \mid k \in \mathbb{Z}, 0<2 k<n\}) .
$$

The integer in $\{2, \ldots, n-1\}$ indicates the codimension of the singular orbit if the normal spaces are real, and the integer in $\{2 k \mid k \in \mathbb{Z}, 0<2 k<n\}$ indicates the codimension of the singular orbit if the normal spaces have constant Kähler angle $\varphi \in(0, \pi / 2)$.

Corollary 4.3. Any singular orbit of a cohomogeneity one action on $\mathbb{C} H^{2}$ is totally geodesic.

Note that by this result we now have a complete classification of the homogeneous hypersurfaces in $\mathbb{C} H^{n}$ for all $n \geq 2$. In view of [9], we call a submanifold $M$ normally homogeneous if $M$ is homogeneous and if the slice representation at $p \in M$ acts transitively on the unit sphere in $\nu_{p} M$. A singular orbit of a cohomogeneity one action is clearly a normally homogeneous submanifold. The above shows that for each $k \in\{2, \ldots, n-1\}$ there exists, up to holomorphic congruence, exactly one normally homogeneous submanifold $F_{k}$ of $\mathbb{C} H^{n}$ with real normal bundle of rank $k$, and for each $k \in\{1, \ldots,[(n-1) / 2]\}$ and each $\varphi \in(0, \pi / 2)$ there exists exactly one, up to holomorphic congruence, normally homogeneous submanifold $F_{k, \varphi}$ of $\mathbb{C} H^{n}$ with normal bundle of rank $2 k$ and constant Kähler angle $\varphi$.

Theorem 4.4. Let $M$ be a homogeneous hypersurface in $\mathbb{C} H^{n}, n \geq 2$. Then $M$ is holomorphically congruent to one of the following hypersurfaces:

(1) a tube of radius $r \in \mathbb{R}_{+}$around the totally geodesic $\mathbb{C} H^{k} \subset \mathbb{C} H^{n}$ for some $k \in\{0, \ldots, n-1\}$;

(2) a tube of radius $r \in \mathbb{R}_{+}$around the totally geodesic $\mathbb{R} H^{n} \subset \mathbb{C} H^{n}$;

(3) a horosphere in $\mathbb{C} H^{n}$;

(4) the minimal ruled real hypersurface $S$ determined by a horocycle in a totally geodesic $\mathbb{R} H^{2} \subset \mathbb{C} H^{n}$, or an equidistant hypersurface to $S$;

(5) a tube of radius $r \in \mathbb{R}_{+}$around the normally homogeneous submanifold $F_{k}$ of $\mathbb{C} H^{n}$ with real normal bundle of rank $k, k \in\{2, \ldots, n-1\}$;

(6) a tube of radius $r \in \mathbb{R}_{+}$around the normally homogeneous submanifold $F_{k, \varphi}$ of $\mathbb{C} H^{n}$ with normal bundle of rank $2 k \in\{2, \ldots, 2[(n-1) / 2]\}$ and constant Kähler angle $\varphi \in(0, \pi / 2)$.

$M=\mathbb{H} H^{n}$ In this case the quaternionic Kähler structure on $\mathbb{H} H^{n}$ induces a quaternionic structure $\mathfrak{J}$ on $\mathfrak{v}$ so that $\mathfrak{v}$ is isomorphic to $\mathbb{H}^{n-1}$ as a (right) quaternionic vector subspace. Let $\mathfrak{v}_{o}$ be a linear subspace of $\mathfrak{v}$ so that $\operatorname{dim}_{\mathbb{R}} \mathfrak{v}_{o}^{\perp} \geq 2$. In [3] the first author and Brück introduced the notion of a quaternionic Kähler angle, which is defined as follows. Let $S^{2}$ be the two-sphere of all almost Hermitian structures in $\mathfrak{J}$. For each nonzero vector $v \in \mathfrak{v}_{o}^{\perp}$ and each $J \in S^{2}$ denote by $\varphi(v, J)$ the Kähler angle of $J v$ and $\mathfrak{v}_{o}^{\perp}$ in the complex vector space $(\mathfrak{v}, J)$. Since $S^{2}$ is compact, 
there exist a minimum and a maximum for these Kähler angles. It was shown in [3] that for each nonzero $v$ there always exists a canonical basis $J_{1}, J_{2}, J_{3}$ of elements in $S^{2}$ (i.e., $J_{\nu} J_{\nu+1}=J_{\nu+2}=-J_{\nu+1} J_{\nu}$, index modulo 3) such that $\varphi\left(v, J_{1}\right)$ is the minimum $\varphi_{1}(v)$ of these Kähler angles and $\varphi\left(v, J_{3}\right)$ is the maximum $\varphi_{3}(v)$ of these Kähler angles. For any canonical basis with this property the Kähler angle $\varphi_{2}(v)=\varphi\left(v, J_{2}\right)$ attains the same value. The triple $\Phi(v)=\left(\varphi_{1}(v), \varphi_{2}(v), \varphi_{3}(v)\right)$ of Kähler angles is called the quaternionic Kähler angle of $\mathfrak{v}_{o}^{\perp}$ with respect to $v$. For a cohomogeneity one action the quaternionic Kähler angle of $\mathfrak{v}_{o}^{\perp}$ must be independent of the choice of the unit vector in $\mathfrak{v}_{o}^{\perp}$. In [3] several examples of subspaces of $\mathbb{H}^{n-1}$ with constant quaternionic Kähler angle were given, but a complete classification is still missing. The examples are as follows:

(a) $\Phi=(0,0,0)$. The linear subspaces of $\mathfrak{v}$ with constant quaternionic Kähler angle $\Phi=(0,0,0)$ are the quaternionic subspaces. A linear subspace $V \subset \mathfrak{v}$ is quaternionic if $J V \subset V$ holds for all $J \in \mathfrak{J}$. For each integer $k$ with $0<k<n$ there exists exactly one (up to orbit equivalence) cohomogeneity one action on $\mathbb{H} H^{n}$ with a singular orbit $F$ of real codimension $4 k$ with the property that the normal spaces of $F$ have constant quaternionic Kähler angle $\Phi=(0,0,0)$, and $F$ is congruent to the totally geodesic $\mathbb{H} H^{n-k} \subset \mathbb{H} H^{n}$.

(b) $\Phi=(0, \pi / 2, \pi / 2)$. The linear subspaces of $\mathfrak{v}$ with constant quaternionic Kähler angle $\Phi=(0, \pi / 2, \pi / 2)$ are the totally complex subspaces. A linear subspace $V \subset \mathfrak{v}$ is totally complex if there exists an almost Hermitian structure $J_{1} \in \mathfrak{J}$ such that $J_{1} V \subset V$ and $J V \subset V^{\perp}$ for all $J \in \mathfrak{J}$ perpendicular to $J_{1}$. For each integer $k \in\{1, \ldots, n-1\}$ there exists exactly one (up to orbit equivalence) cohomogeneity one action on $\mathbb{H} H^{n}$ with a non-totally-geodesic singular orbit $F$ of real codimension $2 k$ with the property that the normal spaces of $F$ have constant quaternionic Kähler angle $\Phi=(0, \pi / 2, \pi / 2)$.

(c) $\Phi=(\pi / 2, \pi / 2, \pi / 2)$. The linear subspaces of $\mathfrak{v}$ with constant quaternionic Kähler angle $\Phi=(\pi / 2, \pi / 2, \pi / 2)$ are the totally real subspaces. A linear subspace $V \subset \mathfrak{v}$ is totally real if $J V \subset V^{\perp}$ holds for all $J \in \mathfrak{J}$. For each integer $k \in$ $\{2, \ldots, n-1\}$ there exists exactly one (up to orbit equivalence) cohomogeneity one action on $\mathbb{H} H^{n}$ with a non-totally-geodesic singular orbit $F$ of real codimension $k$ with the property that the normal spaces of $F$ have constant quaternionic Kähler angle $\Phi=(\pi / 2, \pi / 2, \pi / 2)$.

(d) $\Phi=(0,0, \pi / 2)$. The linear subspaces of $\mathfrak{v}$ with constant quaternionic Kähler angle $\Phi=(0,0, \pi / 2)$ are the 3 -dimensional subspaces of the form ( $\operatorname{Im} \mathbb{H}) v$ for some unit vector $v \in \mathfrak{v}$. There exists exactly one (up to orbit equivalence) cohomogeneity one action on $\mathbb{H} H^{n}$ with a non-totally-geodesic singular orbit $F$ of real codimension 3 with the property that the normal spaces of $F$ have constant quaternionic Kähler angle $\Phi=(0,0, \pi / 2)$.

(e) $\Phi=(\varphi, \pi / 2, \pi / 2), \varphi \in(0, \pi / 2)$. The linear subspaces of $\mathfrak{v}$ with constant quaternionic Kähler angle $\Phi=(\varphi, \pi / 2, \pi / 2), \varphi \in(0, \pi / 2)$, are the linear subspaces with constant Kähler angle $\varphi$ in a totally complex subspace $V$ of $\mathfrak{v}$. Here, the Kähler angle in $V$ is measured with respect to the almost Hermitian structure $J_{1}$ as described in (b). For each integer $k \in\{1, \ldots,[(n-1) / 2]\}$ and each $\varphi \in(0, \pi / 2)$ there exists exactly one (up to orbit equivalence) cohomogeneity one action on $\mathbb{H} H^{n}$ with a non-totally-geodesic singular orbit $F$ of real codimension $2 k$ with the property that the normal spaces of $F$ have constant quaternionic Kähler angle $\Phi=(\varphi, \pi / 2, \pi / 2)$. 
(f) $\Phi=(0, \varphi, \varphi), \varphi \in(0, \pi / 2)$. The linear subspaces of $\mathfrak{v}$ with constant quaternionic Kähler angle $\Phi=(0, \varphi, \varphi), \varphi \in(0, \pi / 2)$, are the complexifications of linear subspaces with constant Kähler angle $\varphi$ in a totally complex subspace $\mathfrak{w}$ of $\mathfrak{v}$. More precisely, let $J_{2} \in \mathfrak{J}$ be an almost Hermitian structure and consider $\mathfrak{v}$ as the complexification of $\mathfrak{w}$ with respect to an almost Hermitian structure $J_{1} \in \mathfrak{J}$ orthogonal to $J_{2}$; that is, $\mathfrak{v}=\mathfrak{w}+J_{1} \mathfrak{w}$ with a $J_{2}$-invariant linear subspace $\mathfrak{w} \subset \mathfrak{v}$. Let $W$ be a linear subspace of the complex vector space $\left(\mathfrak{w}, J_{2}\right)$ with constant Kähler angle $\varphi$. Then the complexification of $W$ with respect to $J_{1}$ is a linear subspace of $\mathfrak{v}$ with constant quaternionic Kähler angle $\Phi=(0, \varphi, \varphi)$. For each integer $k \in\{1, \ldots,[(n-1) / 2]\}$ and each $\varphi \in(0, \pi / 2)$ there exists exactly one (up to orbit equivalence) cohomogeneity one action on $\mathbb{H} H^{n}$ with a non-totally-geodesic singular orbit $F$ of real codimension $4 k$ with the property that the normal spaces of $F$ have constant quaternionic Kähler angle $\Phi=(0, \varphi, \varphi)$.

We conjecture that each cohomogeneity one action on $\mathbb{H} H^{n}$ with a non-totallygeodesic singular orbit is orbit equivalent to one of these examples. This is true for $n=2$, and for the case that the singular orbit has codimension 2 .

Theorem 4.5. The moduli space of all cohomogeneity one actions on $\mathbb{H} H^{2}$ with a non-totally-geodesic singular orbit (up to orbit equivalence) is isomorphic to the set $\{2,3\}$. The number $k \in\{2,3\}$ parametrizes the unique (up to orbit equivalence) cohomogeneity one action on $\mathbb{H} H^{2}$ with a non-totally-geodesic singular orbit of codimension $k$.

Proof. In the case of $\mathbb{H} H^{2}$ the quaternionic vector space $\mathfrak{v}$ has quaternionic dimension one. It is easy to see that every 2-dimensional subspace of a one-dimensional quaternionic subspace has constant quaternionic Kähler angle $\Phi=(0, \pi / 2, \pi / 2)$, and every 3 -dimensional subspace of a one-dimensional quaternionic subspace has constant quaternionic Kähler angle $\Phi=(0,0, \pi / 2)$. The result then follows from (b) and (d) above. Note that codimension 4 occurs for quaternionic Kähler angle $\Phi=(0,0,0)$, which leads to a totally geodesic singular orbit.

Theorem 4.6. The moduli space of all cohomogeneity one actions on $\mathbb{H} H^{n}, n>2$, with a non-totally-geodesic singular orbit with codimension 2 (up to orbit equivalence) is isomorphic to the closed interval $[0, \pi / 2]$. The number $\varphi \in[0, \pi / 2]$ parametrizes the unique (up to orbit equivalence) cohomogeneity one action on $\mathbb{H} H^{n}$ with a non-totally-geodesic singular orbit of codimension 2 for which the normal spaces have constant quaternionic Kähler angle $\Phi=(\varphi, \pi / 2, \pi / 2)$.

Proof. Every 2-dimensional subspace of $\mathfrak{v}$ has constant quaternionic Kähler angle $\Phi=(\varphi, \pi / 2, \pi / 2)$ for some $\varphi \in[0, \pi / 2]$. The result then follows from (b), (c) and (e) above.

$M=\mathbb{O} H^{2}$ In 3 the first author and Brück classified all subspaces $\mathfrak{v}_{o}$ of $\mathfrak{v}=\mathbb{R}^{8}$ for which there exists a subgroup of $K_{x}=\operatorname{Spin}(7)$ that acts transitively on the unit sphere in $\mathfrak{v}_{o}^{\perp}$. In fact, any subspace $\mathfrak{v}_{o}$ of $\mathfrak{v}$ with dimension $k \in\{1,2,4,5,6\}$ has this property, but there are no 3-dimensional subspaces with this property. We denote by $G_{k}^{+}\left(\mathbb{R}^{8}\right)$ the Grassmann manifold of oriented $k$-planes in $\mathbb{R}^{8}$, and by $G_{k}\left(\mathbb{R}^{8}\right)$ the Grassmann manifold of (unoriented) $k$-planes in $\mathbb{R}^{8}$. It is clear that $G_{k}^{+}\left(\mathbb{R}^{8}\right)$ is a two-fold covering of $G_{k}\left(\mathbb{R}^{8}\right)$, and that there is a natural isomorphism between the Grassmann manifolds of $k$ - and $(8-k)$-planes in $\mathbb{R}^{8}$. 
The Lie group $\operatorname{Spin}(7)$ acts on $\mathbb{R}^{8}$ by its irreducible 8-dimensional spin representation. This naturally induces actions of $\operatorname{Spin}(7)$ on $G_{k}^{+}\left(\mathbb{R}^{8}\right)$ and $G_{k}\left(\mathbb{R}^{8}\right)$. For $k=1$, it was proved by Borel [7] that $\operatorname{Spin}(7)$ acts transitively on $G_{1}^{+}\left(\mathbb{R}^{8}\right)=S^{7}$ and that $S^{7}=\operatorname{Spin}(7) / G_{2}$. For $k=2$ we also have a transitive action, so that $G_{2}^{+}\left(\mathbb{R}^{8}\right)=\operatorname{Spin}(7) / U(3)$ (see e.g. [8]), and hence also $G_{6}^{+}\left(\mathbb{R}^{8}\right)=\operatorname{Spin}(7) / U(3)$. Also for $k=3$ the action is transitive, and we have $G_{3}^{+}\left(\mathbb{R}^{8}\right)=\operatorname{Spin}(7) / S O(4)$ (see e.g. [19]), and thus also $G_{5}^{+}\left(\mathbb{R}^{8}\right)=\operatorname{Spin}(7) / S O(4)$.

The action of $\operatorname{Spin}(7)$ on $G_{4}^{+}\left(\mathbb{R}^{8}\right)$ is not transitive, but of cohomogeneity one (see [8] and 13 for details). One singular orbit of this action consists of the so-called Cayley 4-planes in $\mathbb{O}$ introduced by Harvey and Lawson 13 . The submanifolds of (1) all of whose tangent spaces are Cayley 4-planes are so-called Cayley submanifolds of $\mathbb{O}$ and provide a beautiful example of a calibrated geometry. This singular orbit is isomorphic to $\operatorname{Spin}(7) /\left(S U(2)^{3} / \mathbb{Z}_{2}\right)$, and the second singular orbit consists just of the Cayley 4-planes with opposite orientation. This can also be seen in the following way. Let $V \in G_{3}^{+}\left(\mathbb{R}^{8}\right)$ be an oriented 3-plane in $\mathbb{R}^{8}$. We know from the above that $\operatorname{Spin}(7)$ acts transitively on $G_{3}^{+}\left(\mathbb{R}^{8}\right)$ and the isotropy group at $V$ is some $S O(4) \subset \operatorname{Spin}(7)$. There is a unique unit vector $\xi$ in the orthogonal complement $V^{\perp}$ of $V$ in $\mathbb{R}^{8}$ so that the 4-plane $V \oplus \mathbb{R} \xi$ is a Cayley 4-plane. Then $V \oplus \mathbb{R}(-\xi)$ is the same 4-plane with opposite orientation. The action of $S O(4)$ on the unit sphere $S^{4}$ in $V^{\perp}$ is the standard action determined by the two fixed points $\pm \xi$. The principal orbits are the 3 -spheres in $S^{4}$ with center $\xi$. Each such orbit parametrizes in a canonical way a set of oriented 4 -planes in $\mathbb{R}^{8}$ containing the 3 -dimensional subspace $V$. We now turn to the induced action of $\operatorname{Spin}(7)$ on the Grassmannian $G_{4}\left(\mathbb{R}^{8}\right)$ of unoriented 4-planes in $\mathbb{R}^{8}$. This action is clearly of cohomogeneity one as well. The two singular orbits on $G_{4}^{+}\left(\mathbb{R}^{8}\right)$ become identified under the two-fold covering map $G_{4}^{+}\left(\mathbb{R}^{8}\right) \rightarrow G_{4}\left(\mathbb{R}^{8}\right)$ and provide one singular orbit of the action. The second singular orbit in $G_{4}\left(\mathbb{R}^{8}\right)$ is the projection of the principal orbit on $G_{4}^{+}\left(\mathbb{R}^{8}\right)$ containing 4-planes of both orientations. This orbit contains the 4-planes that are constructed from the unique totally geodesic principal orbit of the $S O(4)$-action on $S^{4} \subset V^{\perp}$. The second singular orbit in $G_{4}\left(\mathbb{R}^{8}\right)$ is therefore a 2 -fold subcovering of any principal orbit and thus has the same dimension as the principal orbits.

From Theorem 4.1] it is clear that if the action of $\operatorname{Spin}(7)$ is transitive on $G_{k}\left(\mathbb{R}^{8}\right)$, then all cohomogeneity one actions constructed from a $k$-dimensional subspace of $\mathfrak{v}$ are orbit equivalent. In the case $k=4$, the cohomogeneity one actions induced from a 4-dimensional subspace of $\mathfrak{v}$ up to orbit equivalence are in one-to-one correspondence with the orbits of the action of $\operatorname{Spin}(7)$ on $G_{4}\left(\mathbb{R}^{8}\right)$. Altogether this now implies:

Theorem 4.7. The moduli space of all cohomogeneity one actions on $\mathbb{O} H^{2}$ with a non-totally-geodesic singular orbit (up to orbit equivalence) is isomorphic to the disjoint union

$$
\{2,3,6,7\} \cup(\{4\} \times[0,1]) .
$$

The number $k \in\{2,3,6,7\}$ parametrizes the unique (up to orbit equivalence) cohomogeneity one action on $\mathbb{O} H^{2}$ with a singular orbit of codimension $k$. The set $\{4\} \times[0,1]$ parametrizes the cohomogeneity one actions on $\mathbb{O} H^{2}$ with a singular orbit of codimension 4 (up to orbit equivalence).

The above result says that for each $k \in\{2,3,6,7\}$ there exists exactly one, up to isometric congruence, normally homogeneous submanifold $F_{k}$ of $\mathbb{O} H^{2}$ with normal 
bundle of rank $k$, and for each $\varphi \in[0,1]$ there exists exactly one, up to isometric congruence, normally homogeneous submanifold $F_{4, \varphi}$ of $\mathbb{O} H^{2}$ with normal bundle of rank 4. We now have a complete classification of the homogeneous hypersurfaces in the Cayley hyperbolic plane.

Theorem 4.8. Let $M$ be a homogeneous hypersurface in $\mathbb{O} H^{2}$. Then $M$ is isometrically congruent to one of the following hypersurfaces:

(1) a geodesic hypersphere of radius $r \in \mathbb{R}_{+}$in $\mathbb{O} H^{2}$;

(2) a tube of radius $r \in \mathbb{R}_{+}$around the totally geodesic $\mathbb{O} H^{1} \subset \mathbb{O} H^{2}$;

(3) a tube of radius $r \in \mathbb{R}_{+}$around the totally geodesic $\mathbb{H} H^{2} \subset \mathbb{O} H^{2}$;

(4) a horosphere in $\mathbb{O} H^{2}$;

(5) the minimal homogeneous hypersurface $S$ in $\mathbb{O} H^{2}$, or an equidistant hypersurface to $S$;

(6) a tube of radius $r \in \mathbb{R}_{+}$around the normally homogeneous submanifold $F_{k}$ of $\mathbb{O} H^{2}$ with normal bundle of rank $k, k \in\{2,3,6,7\}$;

(7) a tube of radius $r \in \mathbb{R}_{+}$around the normally homogeneous submanifold $F_{4, \varphi}$ of $\mathbb{O} H^{2}$ with normal bundle of rank 4 and $\varphi \in[0,1]$.

\section{REFERENCES}

1. D. V. Alekseevsky, A. J. Di Scala, Minimal homogeneous submanifolds of symmetric spaces. In: Lie groups and symmetric spaces: in memory of F.I. Karpelevich (Ed. S.G. Gindikin), Amer. Math. Soc. Transl. (2) 210 (2003), 11-25. MR2018350 (2004i:53061)

2. J. Berndt, Homogeneous hypersurfaces in hyperbolic spaces. Math. Z. 229 (1998), 589-600. MR 1664778 (2001c:53065)

3. J. Berndt, M. Brück, Cohomogeneity one actions on hyperbolic spaces. J. Reine Angew. Math. 541 (2001), 209-235. MR.1876290(2002j:53059)

4. J. Berndt, H. Tamaru, Homogeneous codimension one foliations on noncompact symmetric spaces. J. Differential Geom. 63 (2003), 1-40. MR.2015258 (2004k:53076)

5. J. Berndt, H. Tamaru, Cohomogeneity one actions on noncompact symmetric spaces with a totally geodesic singular orbit. Tôhoku Math. J. 56 (2004), 163-177. MR2053317 (2005f:53079)

6. J. Berndt, F. Tricerri, L. Vanhecke, Generalized Heisenberg groups and Damek-Ricci harmonic spaces. Lecture Notes in Mathematics 1598, Springer-Verlag, Berlin, 1995. MR.1340192 (97a:53068)

7. A. Borel, Le plan projectif des octaves et les sphères comme espaces homogènes. C. R. Acad. Sci. Paris 230 (1950), 1378-1380. MR0034768 (11:640c)

8. R. L. Bryant, Submanifolds and special structures on the octonions. J. Differential Geom. 17 (1982), 185-232. MR0664494 (84h:53091)

9. M. Buchner, K. Fritzsche, T. Sakai, Geometry and cohomology of certain domains in the complex projective space. J. Reine Angew. Math. 323 (1981), 1-52. MR0611441 (82k:32030)

10. E. Cartan, Familles de surfaces isoparamétriques dans les espaces à courbure constante. Ann. Mat. Pura Appl. IV. s. 17 (1938), 177-191. MR.1553310

11. A. J. Di Scala, C. Olmos, The geometry of homogeneous submanifolds of hyperbolic space. Math. Z. 237 (2001), 199-209. MR.1836778 (2002d:53064)

12. P. B. Eberlein, Geometry of nonpositively curved manifolds. University of Chicago Press, Chicago, London, 1996. MR 1441541(98h:53002)

13. R. Harvey, H. B. Lawson Jr., Calibrated geometries. Acta Math. 148 (1982), 47-157. MR.0666108 (85i:53058)

14. W.-Y. Hsiang, H. B. Lawson Jr., Minimal submanifolds of low cohomogeneity. J. Differential Geom. 5 (1971), 1-38. MR0298593 (45:7645)

15. K. Iwata, Classification of compact transformation groups on cohomology quaternion projective spaces with codimension one orbits. Osaka J. Math. 15 (1978), 475-508. MR0510490 (80k:57068)

16. K. Iwata, Compact transformation groups on rational cohomology Cayley projective planes. Tôhoku Math. J. (2) 33 (1981), 429-442. MR0643227 (83h:57047) 
17. A. Kollross, A classification of hyperpolar and cohomogeneity one actions. Trans. Amer. Math. Soc. 354 (2002), 571-612. MR1862559 (2002g:53091)

18. T. Levi-Civita, Famiglie di superficie isoparametriche nell'ordinario spazio euclideo. Atti Accad. Naz. Lincei Rend. Cl. Sci. Fis. Mat. Natur. (6) 26 (1937), 355-362.

19. A. L. Onishchik, Inclusion relations among transitive compact transformation groups. Amer. Math. Soc. Transl. (2) 50 (1966), 5-58.

20. B. Segre, Famiglie di ipersuperficie isoparametriche negli spazi euclidei ad un qualunque numero di dimensioni. Atti Accad. Naz. Lincei Rend. Cl. Sci. Fis. Mat. Natur. (6) 27 (1938), 203-207.

21. R. Takagi, On homogeneous real hypersurfaces in a complex projective space. Osaka J. Math. 10 (1973), 495-506. MR0336660(49:1433)

Department of Mathematics, University College, Cork, Ireland

E-mail address: j.berndt@ucc.ie

Department of Mathematics, Hiroshima University, 1-3-1 Kagamiyama, HigashiHiroshima, 739-8526, JAPAN

E-mail address: tamaru@math.sci.hiroshima-u.ac.jp 